\title{
Stars and Bonds in Crossing-Critical Graphs
}

\author{
Petr Hliněný ${ }^{1 *}$ and Gelasio Salazar ${ }^{2}$ \\ ${ }^{1}$ Faculty of Informatics, Masaryk University \\ Botanická 68a, 60200 Brno, Czech Republic \\ e-mail: hlineny@fi.muni.cz \\ ${ }^{2}$ Instituto de Física, Universidad Autónoma de San Luis Potosí \\ San Luis Potosí, Mexico, 78000 \\ e-mail: gsalazar@ifisica.uaslp.mx
}

August 6, 2009

\begin{abstract}
The structure of previous known infinite families of crossing-critical graphs had led to the conjecture that crossing-critical graphs have bounded bandwidth. If true, this would imply that crossing-critical graphs have bounded degree, that is, that they cannot contain subdivisions of $K_{1, n}$ for arbitrarily large $n$. In this paper we prove two new results that revolve around this question. On the positive side, we show that crossing-critical graphs cannot contain subdivisions of $K_{2, n}$ for arbitrarily large $n$. On the negative side, we show that there are simple 3-connected graphs with arbitrarily large maximum degree that are 2 -crossing-critical in the projective plane. Although the former conjecture is now disproved in a subsequent manuscript by Dvořák and Mohar, our results are not affected, and some interesting questions remain. Namely, can the bandwidth conjecture still be true for simple 3-connected graphs in the plane?
\end{abstract}

Keywords: crossing number, crossing-critical graph, bounded degree, bandwidth. 2000 Math Subjects Classification: 05C10, 05C62

\section{Crossing Numbers and Crossing-Critical Graphs}

Unless otherwise stated, throughout this paper our notation and terminology follows Diestel [5].

The crossing number $\operatorname{cr}_{\Sigma}(G)$ of a graph $G$ in a surface $\Sigma$ is the minimum number of pairwise crossings of edges in a drawing of $G$ in $\Sigma$. Whenever the reference to $\Sigma$ is omitted, it is assumed that $\Sigma$ is the plane (or, equivalently in the realm of crossing numbers, the sphere).

Calculating the exact crossing number of a graph is a computationally hard problem, and for many years most crossing number papers focused on calculating or estimating the crossing number of interesting families of graphs. This trend has been reversed in the last few years, as questions of a more structural character have been successfully tackled (see for instance $[2,3$, $10,13,16,19]$.

As with other classical graph theoretical parameters, we gain a great insight into crossing numbers by looking at graphs that are minimal with respect to having a certain crossing number. A graph $G$ is $k$-critical in $\Sigma$ if $\operatorname{cr}_{\Sigma}(G) \geq k$ but $\operatorname{cr}_{\Sigma}(G-e)<k$ for each edge $e$ of $G$. Many

${ }^{*}$ Supported by the Research intent MSM0021622419 of the Czech Ministry of Education. 
interesting questions and results in crossing-critical graphs in the plane are related to the work of Richter and Thomassen [16].

A little over two decades ago, Širáň [18] and Kochol [12] gave nice constructions of crossingcritical graphs. Kochol's family of critical graphs has inspired a good deal of research. These constructions have been generalized by several authors $[17,14,1]$. All such generalizations share one key feature from Kochol's original construction; the infinite families consist of "long and thin" graphs. This led Salazar and Thomas to conjecture that crossing-critical graphs have bounded path-width (see [7]). This conjecture has been proved in [10].

Thomassen observed that all constructions formerly known, including the slightly different flavoured constructions by Hliněný $[9,11]$, satisfy the stronger property of having bounded bandwidth. A graph $G$ has bandwidth at most $k$ if there is a bijection $\beta: V(G) \rightarrow\{1, \ldots,|V(G)|\}$ such that $|\beta(u)-\beta(v)| \leq k$ for each edge $e=u v$ in $G$. This observation was recorded as a conjecture in [15] which we repeat here for the sake of completeness (though it is known to be false nowadays; see Theorem 1.5 and Question 1.6).

Conjecture 1.1 (Disproved in [6].) For each integer $k>0$ there is a number $B(k)$ such that if $G$ is $k$-crossing-critical, then the bandwidth of $G$ is at most $B(k)$.

The following was a weaker and perhaps simpler form of Conjecture 1.1:

Conjecture 1.2 (Disproved in [6].) For each integer $k>0$ there is a number $D(k)$ such that if $G$ is $k$-crossing-critical, then the maximum degree of $G$ is at most $D(k)$.

In this paper we bring two new results formerly inspired by the bounded bandwidth question. From the positive side, we have the following weaker result.

Theorem 1.3 For each integer $k>0$, there is a $f(k)$ such that if $G$ is $k$-crossing-critical, then $G$ does not contain a subdivision of $K_{2, f(k)}$. In particular, $f(k) \leq 30 k^{2}+200 k$.

We also show that the projective-plane version of Conjecture 1.2 is false in a very strong sense even for simple 3-connected graphs and the least interesting value of $k=2$ (we extend this to all nonorientable surfaces in Section 5, at the expense of dropping 3-connectedness).

Theorem 1.4 There is an infinite family of simple 3 -connected graphs $H_{t}, t \geq 4$, such that each $H_{t}$ is 2-crossing-critical in the projective plane and has a vertex of degree $6 t$.

While preparing a revised version of this paper, we have learned about the following surprising new construction, given in [6], which disproves both Conjectures 1.1 and 1.2.

Theorem 1.5 (Dvořák and Mohar [6]) For every $k \geq 171$ and every d, there exists a $k$ crossing-critical graph $H$ containing a vertex of degree at least $d$.

In view of this Theorem 1.5, our Theorem 1.3 now seems to gains even more importance. On the other hand, regarding Theorem 1.4, we remark that the graphs constructed by Dvoŕák and Mohar are not guaranteed to be simple 3-connected (they are using so called "thick edges" which are then replaced with bunches of parallel edges). Therefore, we suggest the following question as an interesting and still open problem worth further research:

Question 1.6 Is Conjecture 1.2 (or 1.1) true when restricted to simple 3-connected graphs G?

The rest of this paper is organized as follows. In Section 2 we study the structure of $\{u, v\}-$ bridges in crossing-critical graphs, where $u, v$ are distinct vertices. This is used in Section 3 to prove Theorem 1.3. In Section 4, we give the construction proving Theorem 1.4. We close with some concluding remarks and questions in Section 5. 


\section{2-cuts and $\{u, v\}$-bridges in crossing-critical graphs}

Recall that a drawing $\mathcal{D}$ of a graph $G$ is a mapping of $G$ into the plane (or the surface $\Sigma$ ), such that the vertices of $G$ are points and the edges are simple curves joining their endvertices. Moreover, it is required that no edge passes through a vertex (except at its ends), every intersection of edges (other than at their endpoints) is a crossing rather than tangential, and no three edges cross in a common point. Throughout this section, we consider graphs drawn in the plane $\mathbb{R}^{2}$ (equivalently, the sphere). We are mostly interested in optimal drawings of a $k$-crossing-critical graph $G$, i.e. in drawings $\mathcal{D}$ of $G$ such that $\operatorname{cr}(\mathcal{D})=\operatorname{cr}(G)$.

To show that no large $K_{2, n}$ subdivisions exist in a $k$-crossing-critical graph ( $k$ is fixed), we first take any two vertices $u, v$, thinking of them as the degree- $n$ vertices in a $K_{2, n}$ subdivision in $G$. We wish to analyze the $\{u, v\}$-bridges in $G$. We use "bridge" in the sense of Tutte: a $U$-bridge in a graph $G$, where $U \subseteq V(G)$, is either a single edge with endvertices in $U$ (a trivial bridge), or a subgraph of $G$ obtained by adding to a connected component $F$ of $G-U$ all the edges attaching $F$ to $U$ together with their ends.

Our major aim in this section is to prove Lemma 2.4, which claims that if $u, v$ are the degree- $n$ vertices of a large $K_{2, n}$ subdivision in $G$, then a large number of $u-v$ paths are drawn (in every optimal drawing of $G$ ) inside a closed disc $\Delta$ bounded by two $u-v$ paths, in such a way that the chunk of $G$ drawn in $\Delta$ is crossing-free and remains connected even after the removal of $u$ and $v$. This is a central prerequisite in the proof of Theorem 1.3.

To prove Lemma 2.4 we need two preliminary results. First we study the implications of a large enough number of $\{u, v\}$-bridges: in an optimal drawing of such a (not necessarily crossing-critical) graph, distinct $\{u, v\}$-bridges are disjointly drawn, and a single face of the drawing is incident with both $u$ and $v$. This is the content of Lemma 2.2. Then we show, in Lemma 2.3, that the number of $\{u, v\}$-bridges is bounded as a function of $k$.

We make essential use of a fundamental result by Richter and Thomassen [16], which proves an intuitively appealing yet elusive fact: $t$-crossing-critical graphs have crossing number bounded by a function of $t$.

Theorem 2.1 (Richter and Thomassen [16]) Every t-crossing-critical graph has crossing number at most $2.5 t+16$.

Our subsequent proofs rely on this upper bound. Any improvement on this bound would immediately imply (marginally, though) sharper bounds in our results.

We start by analyzing drawings with a large number of $\{u, v\}$-bridges. Having a drawing $\mathcal{D}$ of a graph $G$, and a subgraph $H \subseteq G$, we shortly denote by $\mathcal{D}(H)$ the corresponding subdrawing of $H$. We call a face of $\mathcal{D}$ a component of $\mathbb{R}^{2} \backslash \mathcal{D}$.

Lemma 2.2 Let $G$ be a 2-connected graph, and let $u, v$ be different vertices of $G$. Let $t>0$ be an integer. Suppose that $\operatorname{cr}(G) \leq t$, and that $G$ has at least $t+2\{u, v\}$-bridges. Then every optimal drawing $\mathcal{D}$ of $G$ satisfies the following:

(i) No two edges of different $\{u, v\}$-bridges cross each other in $\mathcal{D}$.

(ii) There is a face of $\mathcal{D}$ that is incident with both $\mathcal{D}(u)$ and $\mathcal{D}(v)$.

Proof. Let $G, u, v$, and $t$ be as in the statement of the claim, and let $\mathcal{D}$ be any optimal drawing of $G$. Let $C_{1}, C_{2}, \ldots, C_{s}$ be all the $\{u, v\}$-bridges of $G$, where, by hypothesis, $s \geq t+2$.

First we note that, for each $C_{i}$, the drawing $\mathcal{D}_{i}=\mathcal{D}\left(C_{i}\right)$ of $C_{i}$ induced by $\mathcal{D}$ has a face incident with both $\mathcal{D}_{i}(u)$ and $\mathcal{D}_{i}(v)$. For suppose that some $C_{j}$ does not satisfy this property. 
Since every $C_{i}$ contains a $u-v$ path, then every $C_{i}$ with $i \neq j$ has at least one edge that crosses (in $\mathcal{D}$ ) an edge of $C_{j}$. But then $\mathcal{D}$ has at least $t+1$ crossings, a contradiction. We remark that this observation also implies that if $\mathcal{D}$ satisfies (i), then it must satisfy (ii) as well.

A straightforward corollary of the previous paragraph then implies that, for each $i=$ $1,2, \ldots, s$, there is a drawing $\mathcal{D}_{i}^{\prime}$ of $C_{i}$ such that: (a) $\operatorname{cr}\left(\mathcal{D}_{i}^{\prime}\right)=\operatorname{cr}\left(\mathcal{D}_{i}\right)$; (b) $\mathcal{D}_{i}^{\prime}(u)=(0,1)$ and $\mathcal{D}_{i}^{\prime}(v)=(0,-1)$; and (c) $\mathcal{D}_{i}^{\prime} \backslash\left\{\mathcal{D}_{i}^{\prime}(u), \mathcal{D}_{i}^{\prime}(v)\right\}$ is contained in the interior of the region bounded by the parabolas $x=i\left(1-y^{2}\right)$ and $x=(i+1)\left(1-y^{2}\right)$.

The drawings $\mathcal{D}_{i}^{\prime}$ can then be combined to yield a drawing $\mathcal{D}^{\prime}$ of $G$ that clearly satisfies (i) and (ii). Now (a) in the previous paragraph implies that $\operatorname{cr}\left(\mathcal{D}^{\prime}\right) \leq \operatorname{cr}(\mathcal{D})$. Moreover, (a) also implies that no edges in different $\{u, v\}$-bridges cross each other in $\mathcal{D}$, as otherwise $\mathcal{D}$ would have more crossings than $\mathcal{D}^{\prime}$, contradicting the optimality of $\mathcal{D}$. Thus $\mathcal{D}$ itself satisfies (i). As observed above, this implies that $\mathcal{D}$ also satisfies (ii).

Lemma 2.3 Let $t>0$ be an integer. Suppose that $G$ is a 2 -connected $t$-crossing-critical graph, and let $u, v$ be any two distinct vertices of $G$. Then $G$ has at most $2.5 t+18\{u, v\}$-bridges.

Proof. Let $\mathcal{C}_{u, v}:=\left\{C_{1}, C_{2}, \ldots, C_{s}\right\}$ denote the collection of all $\{u, v\}$-bridges of $G$. We suppose $s \geq 2.5 t+19$, and will show this implies that $\operatorname{cr}(G-e)=\operatorname{cr}(G)$ for some edge $e$ of $G$, thus contradicting the criticality of $G$.

Let $\mathcal{D}$ be an optimal drawing of $G$. Since $\operatorname{cr}(G) \leq 2.5 t+16$ by Theorem 2.1 , then $s \geq$ $2.5 t+19 \geq \operatorname{cr}(G)+3$ and Lemma 2.2 applies. Then it follows from (i) that the number of $\{u, v\}$-bridges that contain an edge crossed in $\mathcal{D}$ is at most $\operatorname{cr}(G)$. So there is a $\{u, v\}$-bridge, say $C_{1}$ without any loss of generality, such that no edge of $C_{1}$ is crossed in $\mathcal{D}$.

Let $\mathcal{D}_{1}$ be any optimal drawing of $G_{1}=G-V\left(C_{1}-\{u, v\}\right.$ ) (or $G_{1}=G-e$ in the case $C_{1}$ is a single edge $e$ ). Note that $\mathcal{C}_{u, v} \backslash\left\{C_{1}\right\}$ is exactly the set of $\{u, v\}$-bridges in $G_{1}$. Since $\operatorname{cr}\left(G-C_{1}\right) \leq \operatorname{cr}(G)$, and so $\left|\mathcal{C}_{u, v} \backslash\left\{C_{1}\right\}\right| \geq 2.5 t+18 \geq \operatorname{cr}\left(G_{1}\right)+2$, then Lemma 2.2 also applies to $G_{1}$. Moreover, for each $i=2,3, \ldots, s, \operatorname{cr}\left(\mathcal{D}\left(C_{i}\right)\right)=\operatorname{cr}\left(\mathcal{D}_{1}\left(C_{i}\right)\right)$ since any strict inequality would imply that $\mathcal{D}$ or $\mathcal{D}_{1}$ is not optimal. By Lemma $2.2(\mathrm{i})$, as applied to both $G$ and $G_{1}$, we get $\operatorname{cr}(\mathcal{D})=\operatorname{cr}\left(\mathcal{D}_{1}\right)$. Thus $\operatorname{cr}(G)=\operatorname{cr}\left(G_{1}\right)$, and hence $\operatorname{cr}(G-e)=\operatorname{cr}(G)$ for each $e \in E\left(C_{1}\right)$.

Lemma 2.4 Let $t, m>0$ be integers. Let $G$ be a 2-connected $t$-crossing-critical graph, and let $\mathcal{D}$ be an optimal drawing of $G$. Suppose that for some vertices $u, v$ of $G$, there are at least $(7.5 t+50) m$ pairwise internally-disjoint $u-v$ paths in $G$. Then there are $m+1$ such $u-v$ paths, say $P_{0}, P_{1}, \ldots, P_{m}$, such that:

(i) $\mathcal{D}\left(P_{0} \cup P_{m}\right)$ bounds a closed disc $\Delta$ containing no crossings of $\mathcal{D}$,

(ii) $\mathcal{D}\left(P_{i}\right) \subseteq \Delta$ for $i=1, \ldots, m-1$, and

(iii) the subgraph of $G-\{u, v\}$ drawn on $\Delta$ is connected, and so $P_{0}, \ldots, P_{m}$ all belong to one $\{u, v\}$-bridge of $G$.

Proof. Let $\mathcal{C}_{u, v}:=\left\{C_{1}, C_{2}, \ldots, C_{s}\right\}$ denote the collection of all $\{u, v\}$-bridges of $G$, and let $p_{i}$ be the cardinality of a maximal set of pairwise internally-disjoint $u-v$ paths in $C_{i}$. Let $r_{i}$ be the number of crossings in $\mathcal{D}$ involving edges of $C_{i}$, with a crossing counting twice if both edges involved are in $C_{i}$.

We first prove that there exists $i \in\{1, \ldots, s\}$ such that $p_{i} \geq m\left(r_{i}+1\right)$. Indeed, seeking a contradiction, suppose that $p_{i} \leq m\left(r_{i}+1\right)-1$ for each $i$. Then, since $\sum_{i=1}^{s} r_{i}=2 \mathrm{cr}(G) \leq 5 t+32$ by Theorem 2.1, and $s \leq 2.5 t+18$ by Lemma 2.3, we can bound the total number of pairwise 


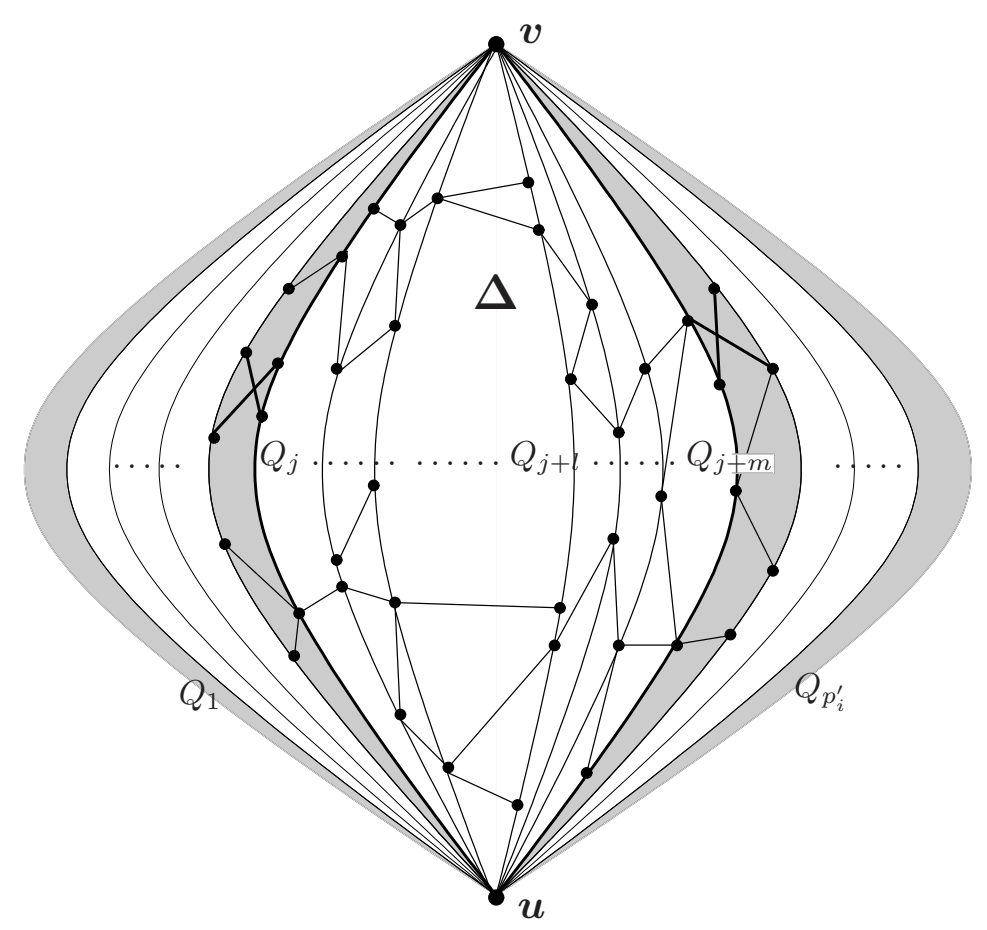

Figure 1: Proof of Lemma 2.4. A collection $Q_{1}, Q_{2}, \ldots, Q_{p_{i}^{\prime}}$ of $u-v$ paths in a $\{u, v\}$-bridge $C_{i}$ of $G$; its bad indices $j-1, j+m$ (Type I) and $p_{i}^{\prime}$ (Type II), depicted with shaded strips, and consecutive $m$ good indices $j, j+1, \ldots, j+m-1$ whose strips form $\Delta$.

internally-disjoint $u-v$ paths in $G$ from above by

$$
\sum_{i=1}^{s} p_{i} \leq \sum_{i=1}^{s}\left(m\left(r_{i}+1\right)-1\right)<m \sum_{i=1}^{s} r_{i}+m s \leq m(5 t+32+2.5 t+18),
$$

contrary to our assumption.

So there is an $i$ such that $p_{i} \geq m\left(r_{i}+1\right)$. Consider a maximum-size collection $Q_{1}, Q_{2}, \ldots, Q_{p_{i}^{\prime}}$ of pairwise internally-disjoint $u-v$ paths in $G$ belonging to $C_{i}$ and not involved in a crossing in $\mathcal{D}$, labeled in agreement with the clockwise cyclic order in which they appear in $\mathcal{D}$ around $u$. See Figure 1. Note that $p_{i}^{\prime} \geq p_{i}-r_{i}$. We say that an index $j \in\left\{1, \ldots, p_{i}^{\prime}\right\}$ is bad if either; (Type I) the closed disc $\Delta^{j}$ bounded by $\mathcal{D}\left(Q_{j} \cup Q_{j+1}\right)$ in the sphere and disjoint from the interior of $Q_{j+2}$ contains a crossing of $\mathcal{D}$, or (Type II) $\Delta^{j}$ contains a face of $\mathcal{D}\left(C_{i}\right)$ incident both with $u$ and $v$ (indices are modulo $p_{i}^{\prime}$ ).

We argue that the number of bad indices is at most $r_{i}+1$. Indeed, as at most $r_{i}$ of the disks $\Delta^{j}$ contain a crossing of $\mathcal{D}$ involving an edge of $C_{i}$, there are at most $r_{i}$ bad indices of Type I. If $\Delta^{j}$ contains a crossing of $\mathcal{D}$, but no one involving $C_{i}$, then $\Delta^{j}$ contains the drawing of another $\{u, v\}$-bridge not crossing $C_{i}$ and so there is a face of $\mathcal{D}\left(C_{i}\right)$ incident both with $u$ and $v$ in $\Delta^{j}$. Finally, there is at most one such face, as otherwise $C_{i}$ would not be a single $\{u, v\}$-bridge. Thus there is at most one bad index of Type II, and therefore at most $r_{i}+1$ bad indices in total.

Now $p_{i}^{\prime} \geq p_{i}-r_{i} \geq(m-1)\left(r_{i}+1\right)+1$, and hence there exist $m$ consecutive good (i.e., not bad) indices $j, j+1, \ldots, j+m-1$ in the cyclic ordering modulo $p_{i}^{\prime}$. Let $\left(P_{0}, \ldots, P_{m}\right):=$ $\left(Q_{j}, \ldots, Q_{j+m}\right)$. It is now straightforward to verify validity of (i), (ii), and (iii) from the definition of a bad index. 


\section{Large bonds in crossing-critical graphs: proof of Theorem 1.3}

In this section we prove Theorem 1.3. We start with a technical dealing with face distance in graph drawings (Lemma 3.1). We then prove Theorem 1.3 for 2-connected graphs (Theorem 3.2), and we finish the section with a proof for the general case.

Let $x, y$ be vertices of a graph $H$, and let $\mathcal{D}$ be a drawing of $H$. A face chain is a sequence of (different) faces of $\mathcal{D}$ such that each consecutive pair of them shares (a segment of) an edge. A face chain joins $x$ and $y$ if it starts from a face incident with $x$ and ends in a face incident with $y$. The face distance $\operatorname{fd}_{\mathcal{D}}(x, y)$ in $\mathcal{D}$ between $x$ and $y$ is the length (i.e. cardinality) of a shortest face chain that joins $x$ and $y$. A subgraph $G$ of $H$ is crossing-free in a drawing $\mathcal{D}$ if no edge of $G$ is crossed in $\mathcal{D}$. Suppose $F$ is an $\{x, y\}$-bridge in $H$, and the subdrawing $\mathcal{D}(F)$ intersects the rest of $\mathcal{D}$ only in $x, y$. A 2-flip of $\mathcal{D}(F)$ at $x, y$ is an operation of cutting-out $\mathcal{D}(F)$ from $\mathcal{D}$ and pasting back the mirror image of it (properly identifying the cutvertices $x, y$ again).

Lemma 3.1 Let $H$ be a planar graph, $x, y \in V(H)$, and $Q_{1}, Q_{2}, Q_{3}$ be three internally disjoint paths connecting $x$ to $y$ in $H$. Suppose $\mathcal{D}_{1}$ is a planar drawing (i.e. without crossings) of $H$, and $\mathcal{D}_{2}$ is an arbitrary drawing of $H$ such that $Q:=Q_{1} \cup Q_{2} \cup Q_{3}$ is drawn crossing-free in $\mathcal{D}_{2}$. Then $\mathrm{fd}_{\mathcal{D}_{2}}(x, y) \geq \mathrm{fd}_{\mathcal{D}_{1}}(x, y)$.

Proof. A standard network-flow duality argument gives that the face distance $\operatorname{fd}_{\mathcal{D}_{1}}(x, y)$ is at least $\ell+1$ if, and only if, there exist $\ell$ pairwise edge-disjoint cycles $C_{1}, \ldots, C_{\ell}$ in $H$ such that each $\mathcal{D}_{1}\left(C_{i}\right), i \in\{1, \ldots, \ell\}$, separates $\mathcal{D}_{1}(x)$ from $\mathcal{D}_{1}(y)$. Note that $Q_{1} \cup Q_{2} \cup Q_{3}$ is a subdivision of the 3 -bond, and thus it is embedded (drawn with no crossings) in the same way under $\mathcal{D}_{1}$ and $\mathcal{D}_{2}$. Fix any $i \in\{1, \ldots \ell\}$. If in $\mathcal{D}_{2}$ there was an arc from $\mathcal{D}_{2}(x)$ to $\mathcal{D}_{2}(y)$ that crossed no edge of $C_{i}$, then clearly there would be a a subpath $P$ of $C_{i}$, with one endpoint in $Q_{j}$ and the other in $Q_{j+1}$ (for some $j \in\{1,2,3\}$, reading the indices of $Q_{j}$ 's modulo 3), internally disjoint from $Q$, such that $P$ crosses $Q_{j+2}$, contradicting that $Q$ is drawn crossing-free under $\mathcal{D}_{2}$. It thus follows that for each $i \in\{1,2, \ldots, \ell\}, \mathcal{D}_{2}\left(C_{i}\right)$ also separates $\mathcal{D}_{2}(x)$ from $\mathcal{D}_{2}(y)$. This is, in turn, a certificate that $\mathrm{fd}_{\mathcal{D}_{2}}(x, y) \geq \ell+1$.

Theorem 3.2 Let $k>0$ be an integer and $G$ be a 2 -connected graph. Suppose that $G$ contains

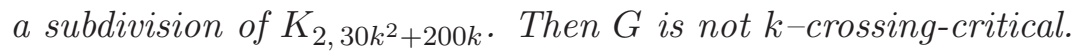

Proof. Let $\mathcal{D}$ be any optimal drawing of $G$. By hypothesis, there are distinct vertices $u, v$ such that there are at least $30 k^{2}+200 k$ pairwise internally disjoint $u-v$ paths in $G$. Setting $m=4 k$, we obtain using Lemma 2.4 a family $P_{0}, \ldots, P_{m}$ of $u-v$ paths satisfying (i)-(iii) in Lemma 2.4. Let $F$ denote the $\{u, v\}$-bridge of $G$ containing all these paths, and $\mathcal{F}:=\mathcal{D}(F)$ be the drawing of $F$ induced by $\mathcal{D}$. Without any loss of generality, the $P_{i}$ 's are labeled according to the clockwise cyclic order in which they appear around $u$ in $\mathcal{F}$.

Let $e$ be the edge of $P_{2 k}$ that is incident with $u$. Note that $e$ cannot be the only edge of $P_{2 k}$, as otherwise $P_{2 k}$ would be a $\{u, v\}$-bridge (of $G$ ) by itself. Let $G_{e}:=G-e$ and $F_{e}:=F-e$. Let $\mathcal{D}_{e}$ be any optimal drawing of $G_{e}$, and let $\mathcal{F}_{e}:=\mathcal{D}_{e}\left(F_{e}\right)$ be the drawing of $F_{e}$ induced by $\mathcal{D}_{e}$.

To complete the proof, we assume $G$ is $k$-crossing-critical, and derive a contradiction. First note that $k$-crossing-criticality of $G$ implies that $\operatorname{cr}\left(\mathcal{F}_{e}\right) \leq \operatorname{cr}\left(\mathcal{D}_{e}\right) \leq k-1<\operatorname{cr}(G)$.

Claim 3.3. To prove the theorem, it suffices to construct a drawing $\overline{\mathcal{F}}$ of $F$ such that

(a) $\operatorname{cr}(\overline{\mathcal{F}}) \leq \operatorname{cr}\left(\mathcal{F}_{e}\right)$,

(b) $\operatorname{fd}_{\overline{\mathcal{F}}}(u, v) \leq \operatorname{fd}_{\mathcal{F}_{e}}(u, v)$. 

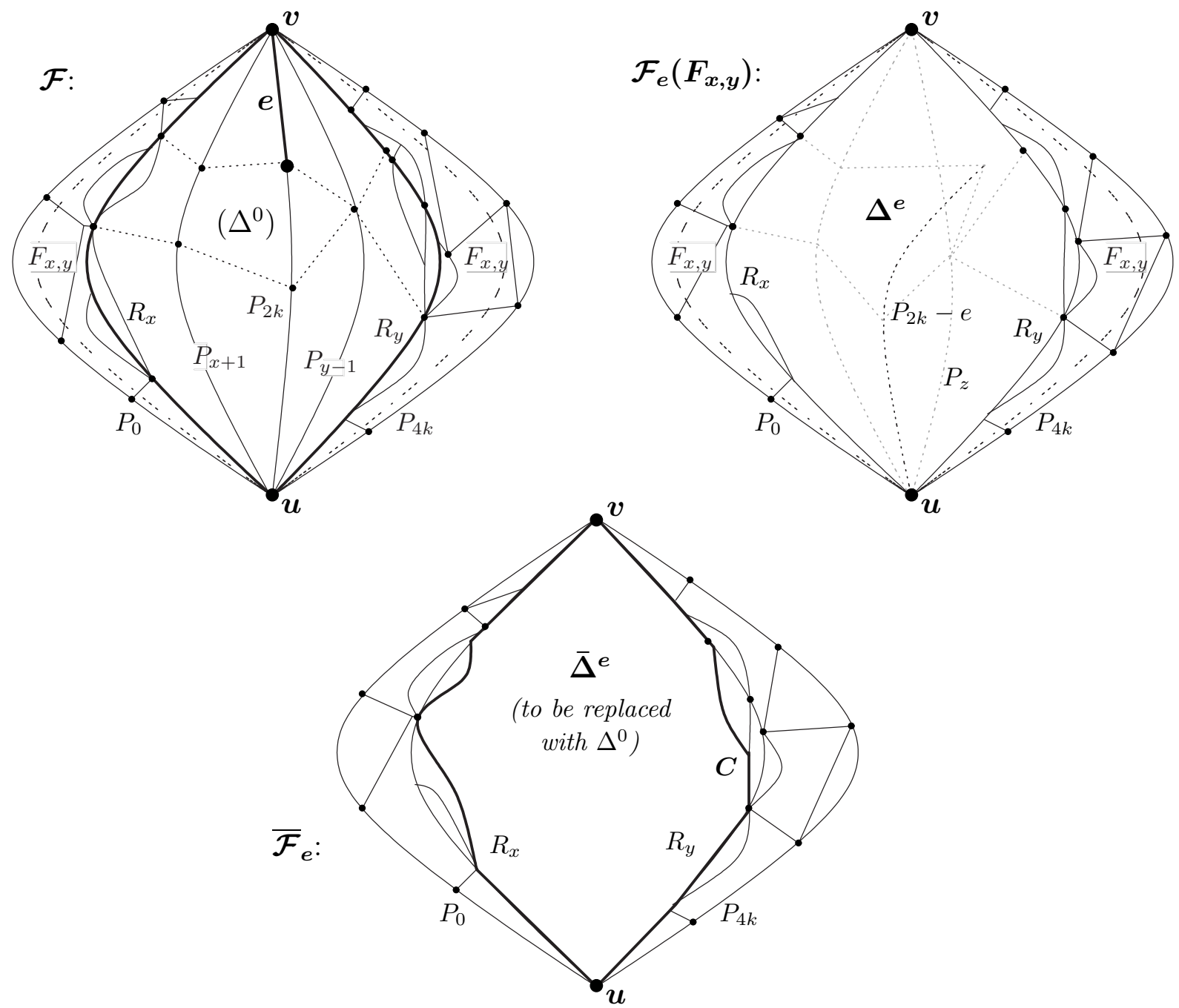

Figure 2: Proof of Theorem 3.2 (Claim 3.3); the drawings $\mathcal{F}, \mathcal{F}_{e}$, and $\overline{\mathcal{F}}_{e}$. The claimed new drawing $\overline{\mathcal{F}}$ results by "replacing" $\bar{\Delta}^{e}$ in $\overline{\mathcal{F}}_{e}$ with the content of $\Delta^{0}$.

Indeed, having such $\overline{\mathcal{F}}$ at hand, we simply replace the subdrawing $\mathcal{F}_{e}$ in $\mathcal{D}_{e}$ with $\overline{\mathcal{F}}$, identifying $\overline{\mathcal{F}}(u)$ with $\mathcal{D}_{e}(u)$ and $\overline{\mathcal{F}}(v)$ with $\mathcal{D}_{e}(v)$. This yields a drawing $\overline{\mathcal{D}}$ of $G$. By (a), there are no more crossings involving two edges of $F$ in $\overline{\mathcal{D}}$ than in $\mathcal{D}_{e}$, and by (b) the replacement operation $\mathcal{F} \mapsto \overline{\mathcal{F}}$ may be performed so that there are no more crossings involving one edge of $F$ and one edge of other $\{u, v\}$-bridge of $G$ (indeed, in any optimal drawing of $G$ every $\{u, v\}$-bridge distinct from $F$ gets drawn along a shortest $u-v$ face chain of the drawing of $F$ ). Hence $\operatorname{cr}\left(\mathcal{D}_{e}\right) \geq \operatorname{cr}(\overline{\mathcal{D}}) \geq \operatorname{cr}(G)$, providing the required contradiction.

It remains to construct $\overline{\mathcal{F}}$ with properties (a) and (b) in Claim 3.3. We start with the drawing $\mathcal{F}$ of $F$, cf. Figure 2 left, and recall from Lemma 2.4 the closed disc $\Delta$ bounded by $\mathcal{F}\left(P_{0} \cup P_{4 k}\right)$ and containing no crossings of $\mathcal{F}$. Without loss of generality, we may assume there is no other $u-v$ path in $F$ internally disjoint from our $P_{0}, \ldots, P_{4 k}$ and drawn under $\Delta$.

For $j \in\{1, \ldots, 2 k-1,2 k+1, \ldots, 4 k-1\}$, let $\mathcal{B}_{j}$ denote the set of those $V\left(P_{j}\right)$-bridges that do not intersect $\left(P_{j-1} \cup P_{j+1}\right)-\{u, v\}$, and let $R_{j}$ denote the subgraph of $F$ induced by $P_{j} \cup\left(\cup \mathcal{B}_{j}\right)$ (informally, we think of $R_{j}$ as $P_{j}$ together with its "local bridges"). Note that $\mathcal{F}\left(R_{j}\right)$ is contained in the disc bounded by $\mathcal{F}\left(P_{j-1}\right) \cup \mathcal{F}\left(P_{j+1}\right) \subseteq \Delta$, and only one face of $\mathcal{F}\left(R_{j}\right)$ contains the rest of the drawing $\mathcal{F}$. Clearly, $V\left(R_{j}\right) \cap V\left(R_{j^{\prime}}\right)=\{u, v\}$ and $E\left(R_{j}\right) \cap E\left(R_{j^{\prime}}\right)=\emptyset$ 
whenever $j \neq j^{\prime}$. We take the minimum index $x \in\{1, \ldots, 2 k-1\}$ and the maximum index $y \in\{2 k+1, \ldots, 4 k-1\}$ such that neither $R_{x}, R_{y}$ are crossed in $\mathcal{F}_{e}$. Such indices exist since at most $2 \operatorname{cr}\left(\mathcal{F}_{e}\right) \leq 2 k-2$ of the $R_{j}$ 's may be involved in a crossing. Furthermore, there exists $z \in\{x+1, \ldots, y-1\} \backslash\{2 k\}$ such that also $P_{z}$ is uncrossed in $\mathcal{F}_{e}$, for the same reason.

Back in the drawing $\mathcal{F}$, let $\Gamma$ be the face of $\mathcal{F}\left(R_{x} \cup R_{y}\right)$ containing $\mathcal{F}(e)$, and let $F_{x, y}$ be the subgraph obtained from $F$ by deleting the vertices drawn in the interior of $\Gamma$. We claim that $F_{x, y}-V\left(R_{x} \cup R_{y}\right)$ has at most two connected components, and if there are exactly two, then one of the components attaches only to $R_{x}$ and the other one only to $R_{y}$. This follows easily since any two such components have to be connected in $F-\{u, v\}$ via a path (since $F$ is a $\{u, v\}$-bridge) intersecting $R_{x-1} \cup R_{y+1}$ by Lemma 2.4 (i). See Figure 2 left.

We are now going to analyze the drawing $\mathcal{F}_{e}\left(F_{x, y}\right)$ of $F_{x, y}$ induced by $\mathcal{F}_{e}$. See Figure 2 right. As neither $R_{x}$ nor $R_{y}$ is crossed in $\mathcal{F}_{e}$, the subdrawing $\mathcal{F}_{e}\left(R_{x} \cup R_{y}\right)$ has a (single) face $\Delta^{e}$ containing $\mathcal{F}_{e}\left(P_{2 k}-e\right)$. In the easy case, $\Delta^{e}$ is also a face of $\mathcal{F}_{e}\left(F_{x, y}\right)$, and then we set $\overline{\mathcal{F}}_{e}=\mathcal{F}_{e}\left(F_{x, y}\right)$ and $\bar{\Delta}^{e}=\Delta^{e}$. Otherwise, by the argument in the previous paragraph, one component $X_{1}$ of $F_{x, y}-V\left(R_{x} \cup R_{y}\right)$ drawn under $\Delta^{e}$ attaches to (say) $R_{x}$, and possibly one such $X_{2}$ attaches to $R_{y}$ (it may even happen that $X_{1}=X_{2}=F_{x, y}-V\left(R_{x} \cup R_{y}\right)$ ). Then we fix an interior point $p \in \mathcal{F}_{e}\left(P_{2 k}-e\right)$, take the drawing $\mathcal{F}_{e}\left(F_{x, y}\right)$, and perform a 2-flip at $u, v$ of the subdrawing induced by $R_{x} \cup X_{1}$. If applicable, we do the same to $R_{y} \cup X_{2}$. Let $\overline{\mathcal{F}}_{e}$ denote the resulting drawing of $F_{x, y}$, and $\bar{\Delta}^{e}$ be the face of $\overline{\mathcal{F}}_{e}\left(R_{x} \cup R_{y}\right)$ containing the point $p$. Now $\bar{\Delta}^{e}$ is also a face of $\overline{\mathcal{F}}_{e}$, as we need.

It follows that the boundary of $\bar{\Delta}^{e}$ in $\overline{\mathcal{F}}_{e}$ forms a cycle $C \subseteq F_{x, y}$ by 2-connectivity, and $C$ consists of two $u-v$ paths $P_{x}^{\prime} \subseteq R_{x}$ and $P_{y}^{\prime} \subseteq R_{y}$ by the definition of $R_{x}, R_{y}$. By invoking a Riemann stereographic projection argument if necessary, we may assume $\bar{\Delta}^{e}$ is a closed disc. See Figure 2 bottom. Let $\Delta^{0}$ be (in the original drawing $\mathcal{F}$ ) the closed disc bounded by $\mathcal{F}(C)$ and containing $\mathcal{F}(e)$, and $F_{0} \subseteq F$ be the graph (thought as "complement" of $F_{x, y}$ ) such that $F_{0} \cap F_{x, y}=C$ and $F_{0} \cup F_{x, y}=F$. Notice that $\mathcal{F}\left(F_{0}\right)$ is drawn crossing-free under $\Delta^{0}$ by Lemma 2.4(i). The final step in the construction of $\overline{\mathcal{F}}$ simply places a suitable homeomorphic copy of $\mathcal{F}\left(F_{0}\right)$ into the face $\bar{\Delta}^{e}$ of $\overline{\mathcal{F}}_{e}$, properly identifying $\mathcal{F}(C)$ with $\overline{\mathcal{F}}_{e}(C)$. Clearly, (a) it holds $\operatorname{cr}(\overline{\mathcal{F}})=\operatorname{cr}\left(\overline{\mathcal{F}}_{e}\right) \leq \operatorname{cr}\left(\mathcal{F}_{e}\right)$.

We finish the proof by showing that $\overline{\mathcal{F}}$ also satisfies (b). Recall that the graphs drawn as $\mathcal{F}_{e}$ and $\overline{\mathcal{F}}-e$ are isomorphic. By the above construction, the subdrawings of $F_{x, y}$ induced by $\mathcal{F}_{e}$ and $\overline{\mathcal{F}}$ are the same up to possible 2 -flips at $u, v$. Particularly, the crossings in $\mathcal{F}_{e}\left(F_{x, y}\right)$ are the same as in $\overline{\mathcal{F}}\left(F_{x, y}\right)$. Transforming every crossing of both these subdrawings $\mathcal{F}_{e}\left(F_{x, y}\right)$ and $\overline{\mathcal{F}}\left(F_{x, y}\right)$ into a degree 4 vertex, $\mathcal{F}_{e}$ and $\overline{\mathcal{F}}$ become new drawings $\mathcal{F}_{e}^{\bullet}$ and $\overline{\mathcal{F} \bullet}$. Then the graphs of $\mathcal{F}_{e}^{\bullet}$ and $\overline{\mathcal{F}^{\bullet}}-e$ are isomorphic, too. Moreover, the drawing $\overline{\mathcal{F}^{\bullet}}$ is crossing-free, i.e. planar, and $\mathcal{F}_{e}^{\bullet}$ has the property that $P_{x} \cup P_{y} \cup P_{z}$ is drawn crossing-free. Thus an application of Lemma 3.1 shows (b) that $\mathrm{fd}_{\mathcal{F}_{e}}(u, v)=\mathrm{fd}_{\mathcal{F}_{e}}(u, v) \geq \mathrm{fd}_{\overline{\mathcal{F} \bullet}-e}(u, v)=\mathrm{fd}_{\overline{\mathcal{F}}-e}(u, v)=\mathrm{fd}_{\overline{\mathcal{F}}}(u, v)$.

It is hardly surprising that Theorem 1.3 follows easily from the $2-$ connected case:

\section{Proof of Theorem 1.3.}

Suppose $G$ is $k$-crossing-critical and contains a subdivision of $K_{2,30 k^{2}+200 k}$. Let $C_{1}, C_{2}$, $\ldots, C_{r}$ be the components of $G$, labeled so that, without any loss of generality, $C_{1}$ contains a subdivision of $K_{2,30 k^{2}+200 k}$. A routine argument shows that each $C_{i}$ is $r_{i}$-crossing-critical for some $r_{i}$ (moreover, $\sum_{i} r_{i}=k$ ). Let $H_{1}, H_{2}, \ldots, H_{s}$ be the blocks of $C_{1}$, labeled so that, without any loss of generality, $H_{1}$ contains a subdivision of $K_{2,30 k^{2}+200 k}$. Again, a routine argument shows that each $H_{i}$ is $s_{i}$-crossing-critical for some $s_{j}$ (moreover, $\sum_{i} s_{j}=r_{1}$ ). In particular, $H_{1}$ is $s_{1}$-crossing-critical for some $s_{1} \leq r_{1} \leq k$, and contains a subdivision of $K_{2,30 k^{2}+200 k}$, and consequently a subdivision of $K_{2,30 s_{1}{ }^{2}+200 s_{1}}$. This contradicts Theorem 3.2. 


\section{Projective critical graphs with large maximum degree: proof of Theorem 1.4}

Throughout this section, crossing numbers refer to drawings in the the projective plane.

We now describe the construction of the graph family $H_{t}, t=1, \ldots$, which for $t \geq 4$ satisfies Theorem 1.4. Our construction and its corresponding analysis have been inspired by the fruitful concept of planar tiles [14]. We, however, turn this classical construction "inside out" to produce a planar belt of planar tiles which we consequently force to "twist" in the projective plane by adding an additional high-degree vertex, connected to both sides of the belt. We refer the reader to Figure 3 for a taste of our construction. This is a projective drawing of $H_{3}$, obtained from three copies of the tile $T^{2}$ (see Figure 4).

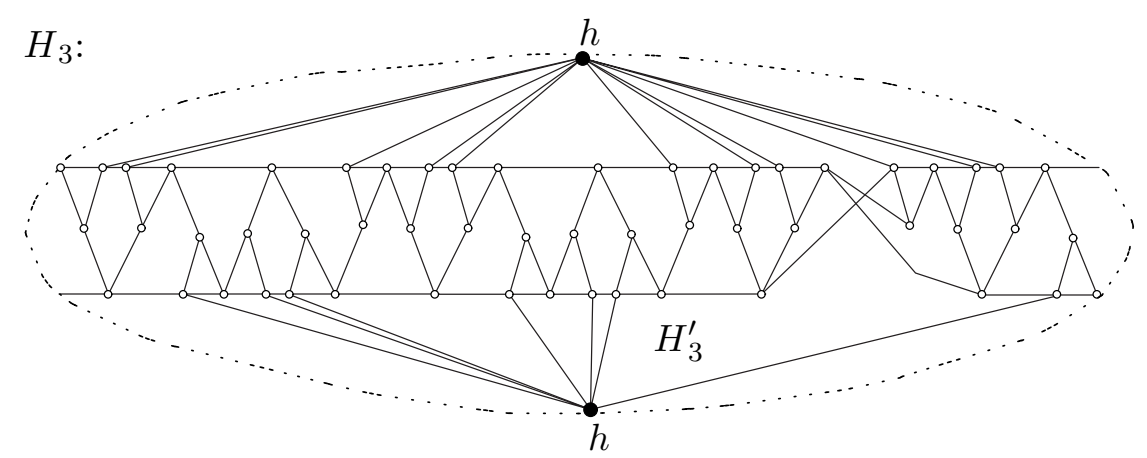

Figure 3: The graph $H_{3}$ drawn in the projective plane (the pairs of opposite points on the dashed ellipse get identified). If vertex $h$ is removed, we obtain $H_{3}^{\prime}$.

The rest of this section is structured as follows. First we give the formal definition of $H_{t}$. Then we show that for every $t \geq 1$ the crossing number of $H_{t}$ minus any edge is at most 1 (Lemma 4.1). Then comes the hardest part-proving that the projective crossing number of $H_{t}$ is at least 2 , for every $t \geq 4$ (Lemma 4.3). The main tool for the latter part is a structural analysis: we examine each edge $e$ of $H_{t}$ and show that, for most choices of $e$, the subgraph $H_{t}-e$ still contains one of the forbidden minors for the projective plane (Figure 7) - and hence the crossing number must be $\geq 1+1$. For the remaining few edges of $H_{t}$, we show that if any two of them cross each other, then the resulting drawing must contain yet another crossing.

\section{Construction of $H_{t}$}

We let $T$ (the tile) denote the graph on 11 vertices and 15 edges depicted in Figure 4 in solid lines, and we distinguish its "boundary" vertices $a, b, c$, and $d$. Let $T^{\prime}$ be a disjoint copy of $T$, and denote by $T^{2}$ the graph obtained from $T \cup T^{\prime}$ by identifying the vertex $c$ of $T$ with vertex $a^{\prime}$ of $T^{\prime}$, and vertex $d$ of $T$ with vertex $b^{\prime}$ of $T^{\prime}$.

Let $T_{1}^{2}, \ldots, T_{k}^{2}$ be $t$ disjoint copies of the graph $T^{2}$, where the boundary vertices of $T_{i}^{2}$ are labeled $a_{i}, b_{i}, c_{i}^{\prime}, d_{i}^{\prime}$. Identify the vertex pair $a_{i+1}, c_{i}^{\prime}$ and the pair $b_{i+1}, d_{i}^{\prime}$, for each $i, 1 \leq i \leq t$ (indices are read modulo $t$, and in the special case $t=1$ identify the vertex pairs $a_{1}, c_{1}^{\prime}$ and $\left.b_{1}, d_{1}^{\prime}\right)$. Denote the resulting graph by $H_{t}^{\prime}$. Thus, $t$ copies of $T^{2}$ are sticked together in a circular fashion to make $H_{t}^{\prime}$. Notice that, unlike in classical crossing-critical constructions, the resulting graph is untwisted ( $H_{t}^{\prime}$ is a planar graph). Now $H_{t}^{\prime}$ is made of $2 t$ copies of the tile $T$. Denote by $p_{i}, q_{i}, r_{i}$, and $p_{i}^{\prime}, q_{i}^{\prime}, r_{i}^{\prime}$, for $i=1, \ldots, t$, the copies of the vertices $p, q, r$ (cf. Figure 4 ) in each $T$. Finally, let $H_{t}$ denote the graph that results by adding a new vertex $h$ to $H_{t}^{\prime}$, and joining $h$ to $p_{i}, q_{i}, r_{i}, p_{i}^{\prime}, q_{i}^{\prime}$, and $r_{i}^{\prime}$ for every $i=1, \ldots, t$. Figure 3 illustrates $H_{3}$. 


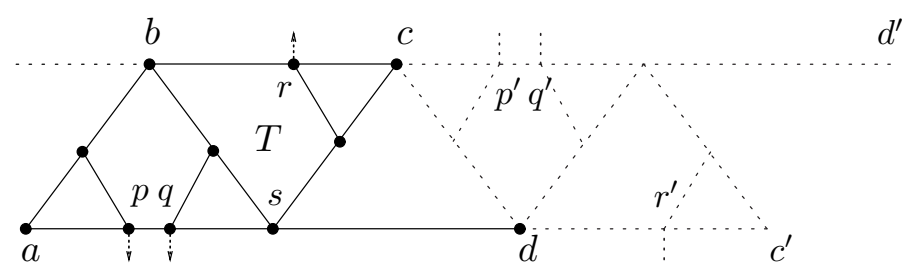

Figure 4: One tile $T$ used in our construction (in solid lines); and another attached copy of $T$ (a scheme in dashed lines), together forming $T^{2}$.

It is clear that every $H_{t}, t \geq 3$, satisfies the connectivity and degree properties claimed in Theorem 1.4. If we aim to show that $H_{t}$ is 2-crossing-critical in the projective plane for each $t \geq 4$, we need to show that (i) $H_{t}$ has a projective crossing number equal to 2 , and that (ii) if we remove any edge from $H_{t}$, then the resulting graph has projective crossing number at most 1. The following statement takes care of (ii) and of the upper-bound part of (i), while subsequent Lemma 4.3 finishes the more difficult direction of (i).

Lemma 4.1 For every $t \geq 1, H_{t}$ can be drawn in the projective plane with at most two crossings. Moreover, for each edge $e \in E\left(H_{t}\right)$, the graph $H_{t}-e$ can be drawn in the projective plane with at most one crossing.

Proof. Figure 3 shows how to draw $H_{3}$ in the projective plane with two crossings. This gets easily extended to a projective drawing of $H_{t}$ with two crossings, for any positive integer $t$. This proves the first statement in the lemma.

We now prove that for any edge $e \in E\left(H_{t}^{\prime}\right), H_{t}^{\prime}-e$ has a projective plane drawing with at most one crossing. We prove this for $t=1$, and simply observe that for $t \geq 2, t-1$ copies of the graph $T^{2}$ can be added to the drawing of $H_{1}^{\prime}-e$ without adding any crossings.

We start with the case $e \in H_{1}^{\prime}$. Consider the natural cylindrical embedding of $H_{1}^{\prime}$, as given in Figure 5.

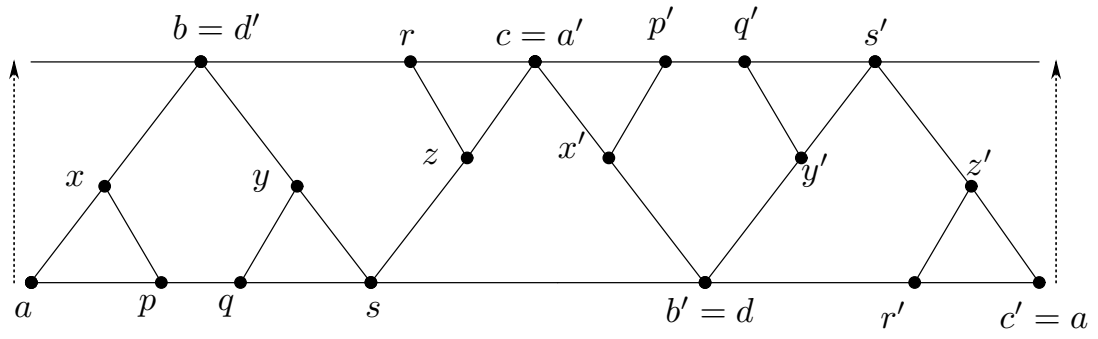

Figure 5: A cylindrical embedding of $H_{1}^{\prime}$.

An edge $e \in E\left(H_{1}^{\prime}\right)$ is of Type $I$ if in this cylindrical embedding there is an arc $\gamma_{e}$ starting at the top rim and ending at the bottom rim, that intersects $e$, and intersects either exactly two other edges (and no vertices), or exactly one vertex (and no edge other than $e$ ). Otherwise, we say that $e$ is of Type II. A quick inspection shows that the only edges of Type II are $p x$ and $p^{\prime} x^{\prime}, q y$ and $q^{\prime} y^{\prime}$, and $r z$ and $r^{\prime} z^{\prime}$.

Suppose first that $e$ is of Type I, and let $\gamma_{e}$ be an arc as above. We cut along $\gamma_{e}$, remove $e$, and twist the resulting band along $\gamma_{e}$, to obtain a Möbius band. If $\gamma_{e}$ only intersects $e$ and a single vertex, then the result is a Möbius band embedding of $H_{1}^{\prime}-e$. If $\gamma_{e}$ intersects two other edges other than $e$, then the loose ends (after cutting along $\gamma_{e}$ ) of these two edges can be rejoined by introducing one crossing; thus in this case we obtain a Möbius band drawing of $H_{1}^{\prime}-e$ with 


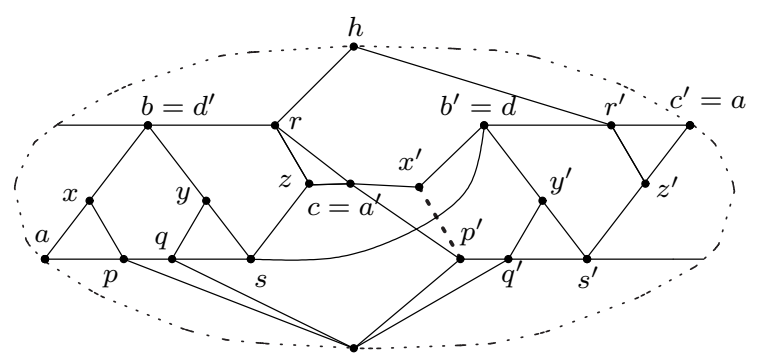

(a) $e=p^{\prime} x^{\prime}$

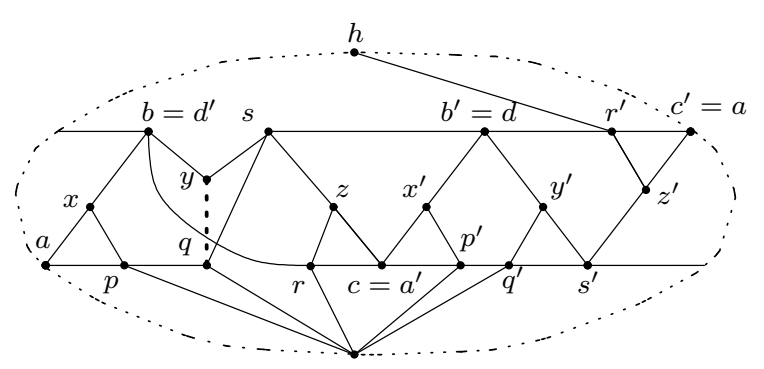

(c) $e=q y$

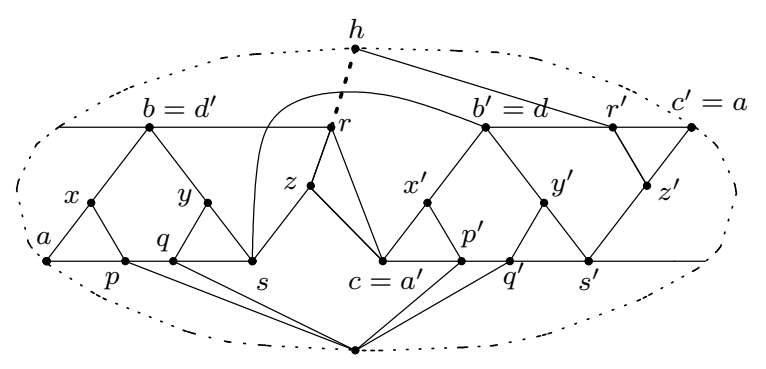

(e) $e=h r$

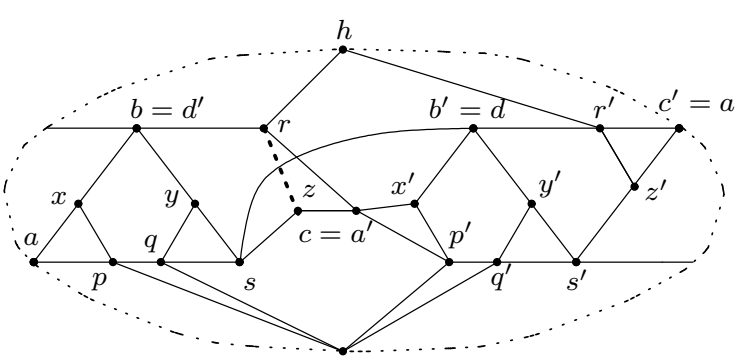

(b) $e=r z$

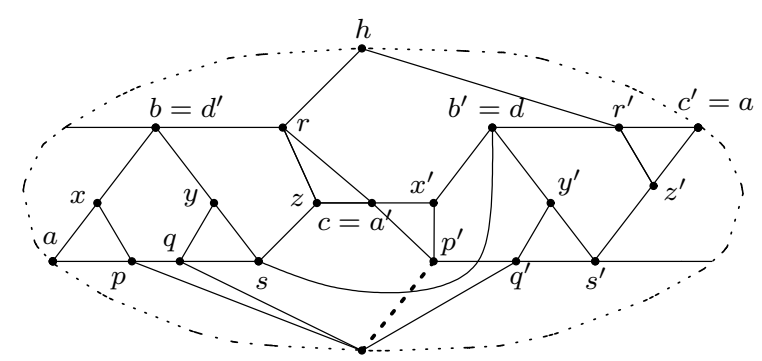

(d) $e=h p^{\prime}$

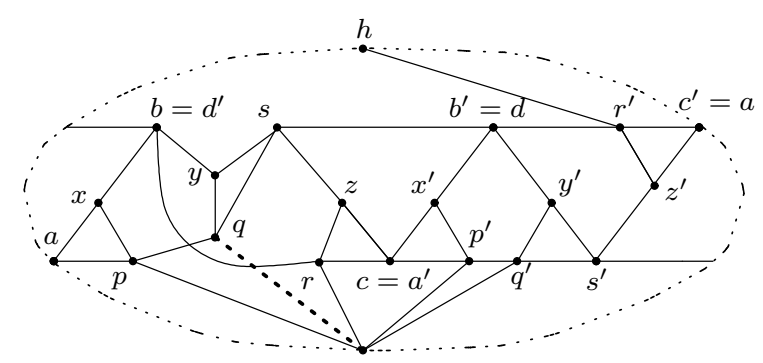

(f) $e=h q$

Figure 6: Drawing $H_{1}-e$ in the projective plane with exactly one crossing. See also Figure 5. In each case, $e$ is indicated as the dashed segment.

exactly one crossing. In either case, we obtain a Möbius band drawing of $H_{1}^{\prime}-e$, with at most one crossing, in which $p, q, r, p^{\prime}, q^{\prime}$, and $r^{\prime}$ are all incident with the Möbius band boundary. By identifying the boundary with a closed disc, we obtain a projective plane drawing of $H_{1}^{\prime}-e$, with at most one crossing, in which $p, q, r, p^{\prime}, q^{\prime}$, and $r^{\prime}$ are all incident with the same face. By this last property, $h$ and its incident edges can be inserted without adding any crossings, thus getting a projective plane drawing of $H_{1}-e$ with at most one crossing.

Thus if $e$ is of Type I, we are done.

Now suppose that $e$ is of Type II, that is, $e \in\left\{p x, p^{\prime} x^{\prime}, q y, q^{\prime} y^{\prime}, r z, r^{\prime} z^{\prime}\right\}$. By symmetry, it suffices to consider the cases $e=p^{\prime} x^{\prime}, e=r z$, and $e=q y$. Figures 6(a), 6(b), and 6(c) show how to draw, respectively, $H_{1}-p^{\prime} x^{\prime}, H_{1}-r z$, and $H_{1}-q y$ with exactly one crossing. This finishes the proof for edges of Type II, and hence for every $e \in E\left(H_{1}^{\prime}\right)$.

Finally, suppose that $e \notin E\left(H_{1}^{\prime}\right)$, that is, $e$ is incident with $h$. By symmetry, it suffices to consider the cases $e=h p^{\prime}, e=h r$, and $e=h q$. Figures 6(d),6(e), and 6(f) show how to draw, respectively, $H_{1}-h p^{\prime}, H_{1}-h r$, and $H_{1}-h q$ with exactly one crossing. This completes the whole proof. 


\section{Projective crossing number of $H_{t}$}

Our final task to prove the criticality of the graphs $H_{t}$ is to show that the projective crossing number of $H_{t}$ is at least (and, in view of Lemma 4.1, equal to) 2.

Recall that an embedding is a drawing with no edge crossings, and that a minor of a graph is obtained from a subgraph by means of contracting edges. We need a result that follows from [8] plus the observation that being embeddable in the projective plane is closed under taking minors.

Lemma 4.2 [8] A graph that contains as a minor either $R_{1}$ or $R_{2}$ (Figure 7) is not embeddable in the projective plane.
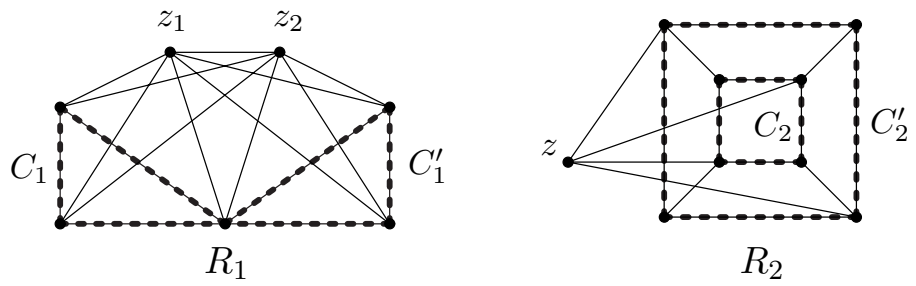

Figure 7: The graphs $R_{1}\left(\simeq K_{7}-C_{4}\right)$ and $R_{2}\left(\simeq K_{4,5}-M_{4}\right)$, two of the "forbidden minors" for the projective plane.

Lemma 4.3 For each $t \geq 4$, the graph $H_{t}$ has projective crossing number at least 2 .

Proof. Let $F \subset E\left(H_{t}\right)$ consist of those edges that are not copies of the edges (in $\left.T\right) e_{p}=p q$, $e_{r}=b r$, or $e_{s}=s d$ (cf. Figure 4).

First we show that (Claim 4.4) if $e \in F$, then $H_{t}-e$ contains $R_{2}$ as a minor. Hence, by Lemma 4.2, the projective crossing number of $H_{t}-e$ is at least 1 , and so such $e$ cannot bear the only crossing of a projective drawing of $H_{t}$. Then we finish the proof with showing that (Claim 4.5), if a crossing in a projective drawing of $H_{t}$ involves two edges not from $F$, then there is another crossing in the drawing.

Claim 4.4. If $e \in F$, then $H_{t}-e$ contains an $R_{2}$ minor.

First, as a warm-up, let us show that $H_{t}$ has a minor isomorphic to $R_{2}$, for all $t \geq 2$. Let $C_{b}$ denote the cycle in $H_{t}^{\prime}$ which results as the union of all copies of the path $b r c p^{\prime} q^{\prime} s^{\prime} d^{\prime} \subset T^{2}$. Let $C_{a}$ similarly denote the union of all copies of the path apqsdr ${ }^{\prime} c^{\prime} \subset T^{2}$ in $H_{t}^{\prime}$. Then $C_{a}, C_{b}$ are disjoint cycles in $H_{t}$, and parts of each of them can be contracted to form the cycles $C_{2}, C_{2}^{\prime}$ of $R_{2}$ (emphasized by thick dots in Figure 7). The vertex $h$ of $H_{t}$ then becomes $z$ in $R_{2}$, and the rest follows easily (see also Figure 3 ).

A more careful consideration of the arguments in the previous paragraph reveals that even the graph $H_{t}-e$ has a minor isomorphic to $R_{2}$ : this is easy if $e \notin E\left(C_{a}\right) \cup E\left(C_{b}\right)$. Otherwise, say for $e \in E\left(C_{a}\right)$, the edge $e$ may be "bypassed" in the cycle by another two edges in $H_{t}^{\prime}$ not incident with $C_{b}$, unless $e$ is a copy of $e_{p}, e_{r}$ or $e_{s}$ above (that is, unless $e \notin F$ ). Thus $H_{t}-e$ contains $R_{2}$ as a minor, as claimed.

Claim 4.5. If there is a crossing in a projective drawing $\mathcal{D}$ of $H_{t}$ involving two edges not from $F$, then $\mathcal{D}$ must contain at least one additional crossing.

Consider an edge crossing $X$ in $\mathcal{D}$ that involves two edges $e_{1}, e_{2} \notin F$, i.e. both $e_{1}, e_{2}$ are copies of $e_{p}, e_{r}$ or $e_{s}$ in $H_{t}$. Let $H_{t}^{\circ}$ denote the graph obtained from $H_{t}$ by subdividing $e_{1}$ and 
$e_{2}$ with new vertices $x, x^{\prime}$, respectively, and let $H_{t}^{\bullet}$ result from $H_{t}^{\circ}$ by identifying $x$ with $x^{\prime}$. In other words, we obtain $H_{t}^{\bullet}$ from $H_{t}$ by turning the crossing $X$ into a new degree- 4 vertex.

Suppose, for a contradiction, that $X$ is the only crossing in $\mathcal{D}$. Hence the corresponding drawing $\mathcal{D}^{\bullet}$ of $H_{t}^{\bullet}$ is crossing-free, and $H_{t}^{\bullet}$ is embeddable in the projective plane. We, however, are going to show that any $H_{t}^{\bullet}$ obtained in the described way must have a minor isomorphic to $R_{1}$ or $R_{2}$; by Lemma 4.2 , this will complete the proof.

Recall the notation from the proof of Claim 4.4. If, say, $e_{1}, e_{2} \in E\left(C_{a}\right)$, then $H_{t}^{\bullet}$ contains a (shorter) circuit $C_{a}^{\prime} \subset C_{a}$, which can be used together with $C_{b}$ to argue that $H_{t}^{\bullet}$ has a minor isomorphic to $R_{2}$ for $t \geq 4$, as above. So we may assume that $e_{1} \in E\left(C_{a}\right)$ and $e_{2} \in E\left(C_{b}\right)$, or vice versa. We divide our analysis into three cases up to symmetry:

(a) $e_{1}$ is a copy of $e_{p}$;

(b) $e_{1}$ is a copy of $e_{r}$ and $e_{2}$ is a copy of $e_{r}$ or $e_{s}$;

(c) both $e_{1}, e_{2}$ are copies of $e_{s}$.
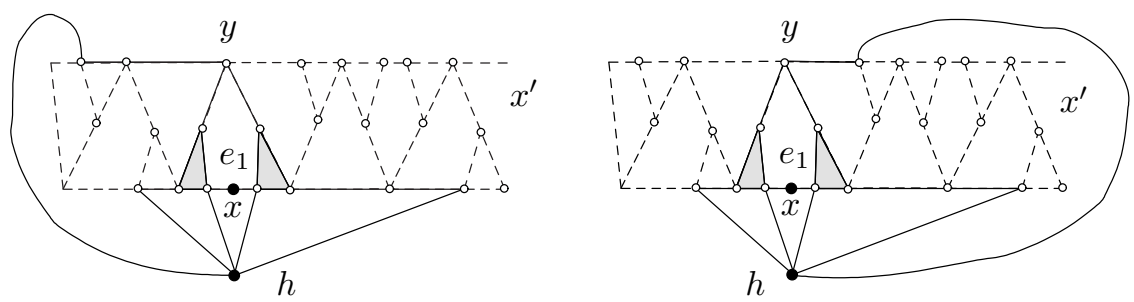

Figure 8: An illustration of Claim 4.5, case (a). $R_{1}$-minors result in both situations.

To deal with (a), we focus on a fragment of the graph $H_{t}^{\circ}$ close to vertex $x$, which is drawn in solid lines in Figure 8. Actually, this case has two similar subcases as in the picture, and in at least one of them none of the solid edges in the picture is incident with the vertex $x^{\prime}$.

Now, in the graph $H_{t}^{\bullet}$, we contract the three solid edges incident with the vertex $y$, and denote by $G_{0}$ the subgraph consisting of the two shaded triangles after the contraction (see Figure 8). Notice that $G_{0}$ together with $h$ and the remaining solid edges forms a subgraph isomorphic to a subdivision of the graph $R_{1}-z_{2}$, where $h$ corresponds to $z_{1}$ and the shaded triangles to the cycles $C_{1}, C_{1}^{\prime}$ in $R_{1}$. Moreover, there is a collection of paths (in dashed edges of $H_{t}^{\bullet}$ ) joining the vertex $x=x^{\prime}$ to all vertices of $G_{0}$, and so $x=x^{\prime}$ is "good for" $z_{2}$ in the graph $R_{1}$. Hence the graph $H_{t}^{\bullet}$ has a minor isomorphic to $R_{1}$.
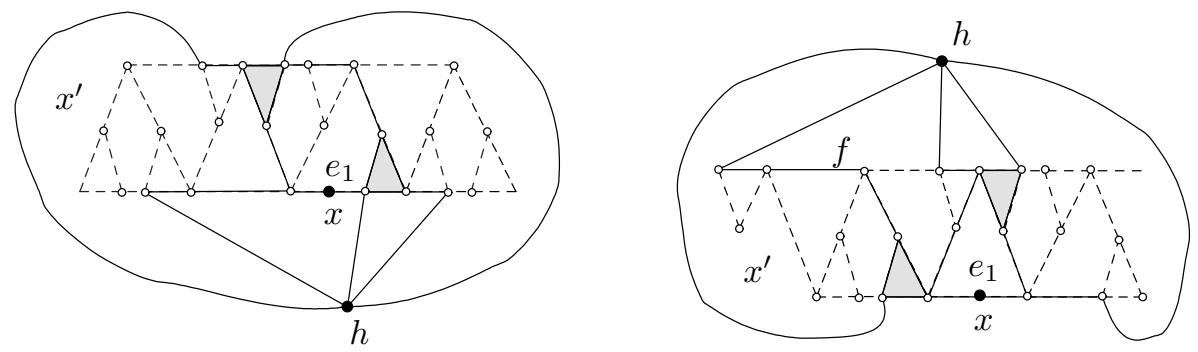

Figure 9: How to get $R_{1}$-minors in the cases (b)-left and (c)-right of Claim 4.5.

We do similarly in the other two cases (b),(c): $e_{1}, e_{2}$ are again chosen without loss of generality such that the subgraphs drawn in solid lines as fragments of $H_{t}^{\circ}$ in Figure 9 are not incident with the vertex $x^{\prime}$. In case (c), if $e_{2}=f$ happens (where $f$ is as in the picture), then we interchange $e_{1}$ and $e_{2}$. Those "solid-line" subgraphs in both cases evidently contain minors 
isomorphic to the graph $R_{1}-z_{2}$, such that the shaded triangles correspond to $C_{1}, C_{1}^{\prime}$ in $R_{1}$. Moreover, the vertex $x=x^{\prime}$ in $H_{t}^{\bullet}$ is connected to vertices of the shaded triangles by five paths, and hence the desired $R_{1}$-minor follows in both cases.

\section{Concluding Remarks}

Since Conjecture 1.2 - the starting point of our research, is now disproved by [6], it appears highly interesting to investigate other structural properties (perhaps stronger than our Theorem 1.3) of $k$-crossing-critical graphs. Given the difficulty of our proofs, this may not be an easy task at all. We note that Theorem 1.3 cannot be strengthened to claim the nonexistence of a $K_{2, f(k)}$-minor: Kochol's 2-crossing-critical family [12] contains arbitrarily large $K_{2, m}$-minors.

On the other hand, our negative Theorem 1.4 gets straightforwardly generalized to other nonorientable surfaces if we do not insist on the 3-connectivity property.

Theorem 5.1 For any $t \geq 4$ and $m \geq 1$, there exists a graph $G_{m, t}$ that is 2-crossing-critical in the nonorientable surface $\mathcal{N}_{m}$ of genus $m$, and has a vertex of degree $6 t$.

Proof. Let $F$ denote a graph embeddable into the projective plane such that $F$ is 2-crossingcritical in the ordinary plane, and such that $F-e$ is nonplanar for every edge $e$ of $F$ ( $K_{6}$ minus an edge is an example of such an $F$ ). We construct the graph $G_{m, t}$ for $m>1$ as the disjoint union of $H_{t}$ (any $H_{t}$ satisfying Theorem 1.4) and $m-1$ copies of $F$.

We claim that $G_{m, t}-e$ has crossing number at most one in $\mathcal{N}_{m}$, for any edge $e$ of $G_{m, t}$. For if $e$ is in $H_{t}$, then one crosscap may be used to draw $H_{t}-e$ with $\leq 1$ crossing, and the remaining crosscaps allow us to embed the $m-1$ copies of $F$. On the other hand, if $e$ is in a copy $F_{1}$ of $F$, then one can combine a planar drawing of $F_{1}-e$ having a single crossing with $m-2$ projective-planar embeddings of $F$ and an embedding of $H_{t}$ along the remaining two crosscaps (the projective-planar drawing in Figure 3 can be turned into a 2-crosscap embedding by introducing a crosscap through the common endvertex of two crossed edges). Thus, in any case, $G_{m, t}-e$ has crossing number at most one in $\mathcal{N}_{m}$, as claimed.

It remains to show that $G_{m, t}$ cannot be drawn into $\mathcal{N}_{m}$ with only one crossing. By way of contradiction, suppose that such a drawing $\mathcal{D}$ exists. We need to analyze two cases separately.

First, suppose that the only crossing in $\mathcal{D}$ involves two edges in $H_{t}$. Then each copy of $F$ is embedded in $\mathcal{N}_{m}$, and therefore contains a noncontractible cycle. Cutting along these $m-1$ disjoint noncontractible cycles leaves a (projective-planar) drawing that contains a (projectiveplanar) drawing of $H_{t}$ with exactly 1 crossing, contradicting that $H_{t}$ is 2 -crossing-critical in the projective plane.

Second, suppose that the only crossing in $\mathcal{D}$ involves at least an edge $e$ in a copy $F_{1}$ of $F$. Thus removing $e$ leaves an embedding of the disjoint union of the other copies of $F$, and of $F_{1}-e$ and $H_{t}$. Analogously to the previous paragraph, each of the other copies of $F$, as well as (nonplanar) $F_{1}-e$, contain a noncontractible cycle, and cutting along these $m-1$ disjoint cycles yields an embedding of $H_{t}$ in the projective plane, again contradicting that $H_{t}$ is 2 -crossing-critical in the projective plane.

The above construction can be easily modified (by performing 1-sums on the connected components of $G_{m, t}$ ) to yield a connected graph with $m-1$ vertex 1 -cuts. This brings us back to Question 1.6, generalized to arbitrary, orientable and nonorientable, surfaces: so far, we can only answer (negatively) this question for the projective plane, for the special case $k=2$.

The proof above also illustrates the issues involved in a related open problem posed by DeVos, Mohar, and Śámal [4]: If $G$ is the disjoint union of the (say blue) graph $G_{1}$ and the 
(say red) graph $G_{2}$, is it true that an optimal drawing of $G$ always exists that has no red-blue crossing? A positive answer to this would allow us to apply the principle of the construction in Theorem 5.1 more generally; in particular, we could then extend Dvořák-Mohar's example in Theorem 1.5 to all (orientable) surfaces.

\section{Acknowledgements}

We thank the two anonymous referees for several suggestions that greatly improved the presentation of this paper.

\section{References}

[1] D. Bokal, Infinite families of crossing-critical graphs with prescribed average degree and crossing number. Preprint (2006).

[2] D. Bokal, On the crossing numbers of Cartesian products with paths. J. Comb. Theory, Ser. B 97 (2007), 381-384.

[3] D. Bokal, G. Fijavz, B. Mohar, The Minor Crossing Number. SIAM J. Discrete Mathematics 20 (2006), 344-356.

[4] M. DeVos, B. Mohar, R. Šámal, Unexpected behavior in crossing sequences. Manuscript (2008).

[5] R. Diestel, Graph theory, Graduate Texts in Mathematics 173, Springer-Verlag, New York 1997, 2000, 2006.

[6] Z. Dvořák and B. Mohar, Crossing-critical graphs with large maximum degree. Manuscript (2009).

[7] J. Geelen, B. Richter, G. Salazar, Embedding grids in surfaces. Europ. J. Combinatorics 25 (2004), 785-792.

[8] H. Glover, J.P. Huneke, C.S. Wang, 103 Graphs That Are Irreducible for the Projective Plane. J. of Comb. Theory Ser. B 27 (1979), 332-370.

[9] P. Hliněný, Crossing-critical graphs and path-width, In: Graph Drawing GD 2001, Vienna Austria, September 2001. Lecture Notes in Computer Science 2265, Springer Verlag 2002, $102-114$.

[10] P. Hliněný, Crossing-critical graphs have bounded path-width. J. of Combinatorial Theory ser. B 88 (2003), 347-367.

[11] P. Hliněný, New infinite families of almost-planar crossing-critical graphs. Electronic Journal of Combinatorics 15 (2008), \#R102.

[12] M. Kochol, Construction of crossing-critical graphs. Discrete Math. 66 (1987), 311-313.

[13] J. Leaños and G. Salazar, On the additivity of the crossing numbers of graphs. Journal of Knot Theory and its Ramifications, in press (2008).

[14] R.B. Richter, B. Pinontoan, Crossing Numbers of Sequences of Graphs II: Planar Tiles. Journal of Graph Theory 42 (2003), 332-341. 
[15] R.B. Richter, G. Salazar, A Survey of Good Crossing Number Theorems and Questions, to appear, 2008.

[16] R.B. Richter and C. Thomassen, Minimal graphs with crossing number at least $k$. J. Combinatorial Theory Ser. B 58 (1993), 217-224.

[17] G. Salazar, Infinite families of crossing-critical graphs with given average degree. Discrete Math. 271 (2003), 343-350.

[18] J. Širán̆, Infinite families of crossing-critical graphs with a given crossing. number. Discrete Math. 48 (1984), 129-132.

[19] D.R. Wood and J.A. Telle, Planar Decompositions and the Crossing Number of Graphs with an Excluded Minor. Lecture Notes in Computer Science 4372, 150-161. Springer, 2007. 